\title{
MINIMUM DISTANCE ESTIMATOR FOR A HYPERBOLIC STOCHASTIC PARTIAL DIFFERENTIAL EQUATION
}

Abstract. We study a minimum distance estimator in $L_{2}$-norm for a class of nonlinear hyperbolic stochastic partial differential equations, driven by a two-parameter white noise. The consistency and asymptotic normality of this estimator are established under some regularity conditions on the coefficients. Our results are applied to the two-parameter Ornstein-Uhlenbeck process.

1. Introduction. In recent years, there has been a growing interest in parameter estimation based on the minimum distance technique. For instance, Dietz and Kutoyants $(1992,1997)$ studied the problem of estimation of a parameter by the observations of an ergodic diffusion process. The Ornstein-Uhlenbeck process with small diffusion coefficients was treated by Kutoyants (1994), Kutoyants et al. (1994), Kutoyants and Pilibossian (1994) and Hénaff (1995). Models for random field diffusions were considered by Kutoyants and Lessi (1995) for the distance defined by Hilbert-type metrics.

The purpose of this paper is to extend their results to a more general class of random fields. More precisely, we deal with the following nonlinear hyperbolic stochastic partial differential equation: for any $\left(t^{1}, t^{2}\right) \in \mathbb{R}_{+}^{2}$,

$$
\begin{aligned}
\frac{\partial^{2} X_{t^{1}, t^{2}}}{\partial t^{1} \partial t^{2}}= & S^{1}\left(\theta_{0}, t^{1}, t^{2}\right) \frac{\partial X_{t^{1}, t^{2}}}{\partial t^{2}}+S^{2}\left(\theta_{0}, t^{1}, t^{2}\right) \frac{\partial X_{t^{1}, t^{2}}}{\partial t^{1}} \\
& +S^{3}\left(\theta_{0}, t^{1}, t^{2}, X\right)+\varepsilon \dot{W}_{t^{1}, t^{2}},
\end{aligned}
$$

with the initial condition $X_{t^{1}, t^{2}}=x$ on the axes, $x \in \mathbb{R}$.

2000 Mathematics Subject Classification: 62M09, 62F12.

Key words and phrases: minimum distance estimator; random fields; small noise; stochastic partial differential equations. 
The coefficients are mesurable functions

$$
\begin{aligned}
& S^{i}: \Theta \times\left[0, T^{1}\right] \times\left[0, T^{2}\right] \rightarrow \mathbb{R}, \quad i=1,2, \\
& S^{3}: \Theta \times\left[0, T^{1}\right] \times\left[0, T^{2}\right] \times \mathcal{C} \rightarrow \mathbb{R},
\end{aligned}
$$

where $\Theta \subset \mathbb{R}^{k}$ and $\mathcal{C}$ stands for the set of all continuous real-valued functions defined on $\left[0, T^{1}\right] \times\left[0, T^{2}\right] . \quad\left\{\dot{W}_{t^{1}, t^{2}}:\left(t^{1}, t^{2}\right) \in\left[0, T^{1}\right] \times\left[0, T^{2}\right]\right\}$ is a onedimensional two-parameter white noise. Equations of this kind appear, for example, in the problem of constructing a Wiener sheet on manifolds (see Norris (1995)) and in nonlinear filtering theory for two-parameter processes (see Korezlioglu et al. (1983)). Their solutions are called two-parameter diffusion processes and there are two different approaches to solving them. The first one was introduced by Farré and Nualart (1993). By a solution they mean a random field $\left\{X_{t^{1}, t^{2}}:\left(t^{1}, t^{2}\right) \in\left[0, T^{1}\right] \times\left[0, T^{2}\right]\right\}$ adapted to the natural filtration associated with the Wiener sheet $W$ and satisfying

$$
\begin{aligned}
X_{t^{1}, t^{2}}= & x+\int_{0}^{t^{1}} \int_{0}^{t^{2}} S^{1}\left(\theta_{0}, s^{1}, s^{2}\right) X\left(s^{1}, d s^{2}\right) d s^{1} \\
& +\int_{0}^{t^{2}} \int_{0}^{t^{1}} S^{2}\left(\theta_{0}, s^{1}, s^{2}\right) X\left(d s^{1}, s^{2}\right) d s^{2} \\
& +\int_{0}^{t^{1}} \int_{0}^{t^{2}} S^{3}\left(\theta_{0}, s^{1}, s^{2}, X\right) d s^{1} d s^{2}+\varepsilon W_{t^{1}, t^{2}} .
\end{aligned}
$$

The other one is due to Rovira and Sanz-Solé (1995, 1996) who used a method based on the Green function $\gamma_{t^{1}, t^{2}}\left(\theta_{0}, s^{1}, s^{2}\right)$ associated with the second order differential operator

$\mathcal{L} f\left(t^{1}, t^{2}\right)=\frac{\partial^{2} f}{\partial t^{1} \partial t^{2}}\left(t^{1}, t^{2}\right)-S^{1}\left(\theta_{0}, t^{1}, t^{2}\right) \frac{\partial f}{\partial t^{2}}\left(t^{1}, t^{2}\right)-S^{2}\left(\theta_{0}, t^{1}, t^{2}\right) \frac{\partial f}{\partial t^{1}}\left(t^{1}, t^{2}\right)$.

Note that $\gamma_{t^{1}, t^{2}}\left(\theta_{0}, s^{1}, s^{2}\right)$ is the solution to the partial differential equation

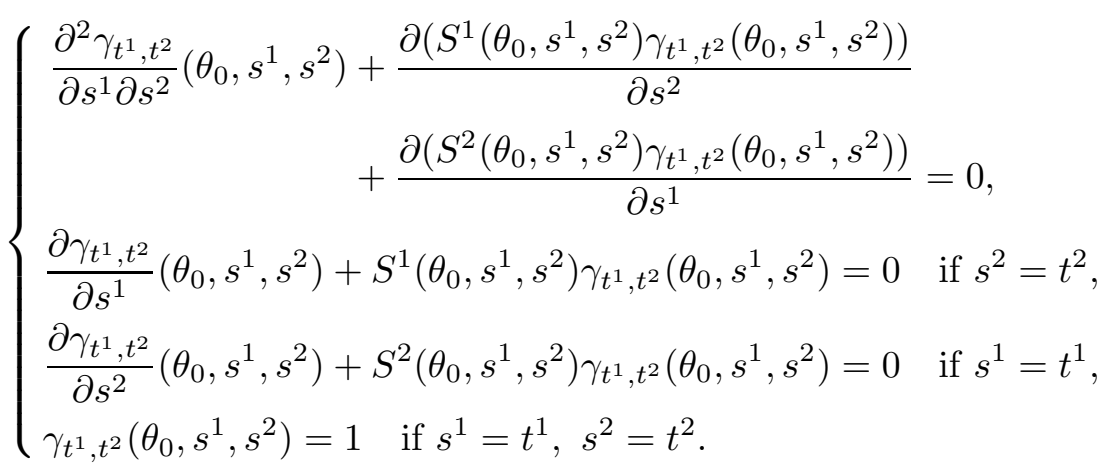


The solution to (1) is defined by

$$
\begin{aligned}
X_{t^{1}, t^{2}}= & x+\int_{0}^{t^{1}} \int_{0}^{t^{2}} \gamma_{t^{1}, t^{2}}\left(\theta_{0}, s^{1}, s^{2}\right) S^{3}\left(\theta_{0}, s^{1}, s^{2}, X\right) d s^{1} d s^{2} \\
& +\varepsilon \int_{0}^{t^{1}} \int_{0}^{t^{2}} \gamma_{t^{1}, t^{2}}\left(\theta_{0}, s^{1}, s^{2}\right) W\left(d s^{1}, d s^{2}\right) .
\end{aligned}
$$

These two apparently different ways of solving equation (1) can be shown to be equivalent (see Rovira and Sanz-Solé 1996, Proposition 2.4).

Now, we state the problem. The coefficients $S^{i}$ are supposed to be known but the value of the parameter $\theta_{0}$ is unknown. Our aim is to estimate $\theta_{0}$ by an $L_{2}$-minimum distance estimator $(\mathrm{MDE}) \theta_{\varepsilon}^{*}$. The case $S^{2}=0$ was treated by Kutoyants and Lessi (1995).

We define $\theta_{\varepsilon}^{*}$ by

$$
\theta_{\varepsilon}^{*}=\arg \inf _{\theta \in \Theta}\|X-x(\theta)\|_{L^{2}(\mu)},
$$

which means that $\theta_{\varepsilon}^{*}$ is a solution to the equation

$$
\left\|X-x\left(\theta_{\varepsilon}^{*}\right)\right\|_{L^{2}(\mu)}=\inf _{\theta \in \Theta}\|X-x(\theta)\|_{L^{2}(\mu)}
$$

where $\|\cdot\|_{L^{2}(\mu)}$ denotes the $L^{2}(\mu)$-norm associated with a finite measure $\mu$ and $\left\{x_{t^{1}, t^{2}}(\theta):\left(t^{1}, t^{2}\right) \in\left[0, T^{1}\right] \times\left[0, T^{2}\right]\right\}$ is the solution of equation (1) when $\theta_{0}$ is replaced by $\theta$ and $\varepsilon=0$. Let us remark that $x_{t^{1}, t^{2}}(\theta)$ is a deterministic function.

The rest of the paper is organized as follows. In Section 2, we introduce notations and state some conditions on the coefficients which will be used throughout. We also recall some properties of the Green function $\gamma_{t^{1}, t^{2}}\left(\theta_{0}, s^{1}, s^{2}\right)$ and give some preliminary lemmas. Section 3 is devoted to the study of the asymptotic behavior of $\theta_{\varepsilon}^{*}$ as $\varepsilon \rightarrow 0$ through its consistency and asymptotic normality.

As usual, all constants appearing in the proofs are called $C$, although they may vary from one occurrence to another.

2. Notations and preliminaries. Let $\mathbb{R}_{+} i=[0, \infty)$ and $T=\left(T^{1}, T^{2}\right) \in$ $\mathbb{R} R_{t}$ stands for the rectangle $\left[0, t^{1}\right] \times\left[0, t^{2}\right]$. The set $\Theta$ of the parameters is a bounded open subset of $\mathbb{R}^{k}$, and $\varepsilon \in(0,1]$.

Now, we state the conditions on the coefficients.

- (H1) For any $\theta \in \Theta, S^{i}(\theta, \cdot), i=1,2$, is uniformly bounded and has uniformly bounded derivatives. 
- (H2) There exists a constant $C>0$ such for that for any $(x, y) \in \mathcal{C} \times \mathcal{C}$, $t \in R_{T}$ and $\theta \in \Theta$,

$$
\begin{aligned}
\left|S^{3}(\theta, t, x)-S^{3}(\theta, t, y)\right| & \leq C\left|x_{t}-y_{t}\right|, \\
\left|S^{3}(\theta, t, x)\right| & \leq C\left(1+\left|x_{t}\right|\right) .
\end{aligned}
$$

- (H3) For any $t \in R_{T}, S^{i}(\cdot, t), i=1,2$, has uniformly bounded first order and mixed second order partial dervatives.

Let $\dot{S}_{\theta}^{3}$ denote the vector function of the derivatives of $S^{i}, i=1,2$, with respect to $\theta$ :

$$
\dot{S}_{\theta}^{i}(\theta, t)=\left(\frac{\partial S^{i}}{\partial \theta^{1}}(\theta, t), \ldots, \frac{\partial S^{i}}{\partial \theta^{k}}(\theta, t)\right)^{\prime}
$$

where $A^{\prime}$ stands for the transpose of the matrix $A$.

If $S^{3}(\theta, t, x)=S^{3}\left(\theta, t, x_{t}\right)$, we denote by $\dot{S}_{\theta}^{3}\left(\theta, t, x_{t}\right)$ the derivative of $S^{3}$ in $x$, i.e. $\dot{S}_{\theta}^{3}\left(\theta, t, x_{t}\right)=\left.\frac{\partial S^{3}}{\partial x}(\theta, t, x)\right|_{x=x_{t}}$. We let $\dot{S}_{\theta}^{3}\left(\cdot, t, x_{t}\right)$ be the vector function of the derivatives of $S^{3}(\cdot, t, x)$ in $\theta$.

- (H4) For any $x \in \mathcal{C}, \theta \in \Theta$ and $t \in R_{T}$, we have $S^{3}(\theta, t, x)=S^{3}\left(\theta, t, x_{t}\right)$ and:

(i) $S^{3}(\theta, t, \cdot)$ is differentiable with uniformly bounded derivatives, $\dot{S}_{x}^{3}(\theta, t, \cdot)$ is continuous and there exist constants $C>0, a \in(0,1]$ and $b \in(0,1]$ such that for any $(x, y) \in \mathcal{C} \times \mathcal{C},\left(\theta_{1}, \theta_{2}\right) \in \Theta^{2}$ and $t \in R_{T}$,

$$
\left|\dot{S}_{x}^{3}\left(\theta_{1}, t, x_{t}\right)-\dot{S}_{x}^{3}\left(\theta_{2}, t, y_{t}\right)\right| \leq C\left(\left|x_{t}-y_{t}\right|^{a}+\left|\theta_{1}-\theta_{2}\right|^{b}\right) ;
$$

(ii) $S^{3}\left(\cdot, t, x_{t}\right)$ is differentiable, $\dot{S}_{\theta}^{3}\left(\cdot, t, x_{t}\right)$ is continuous and for any compact subset $\mathcal{K}$ of $\mathbb{R}$ there exist constants $C_{\mathcal{K}}>0, c \in(0,1]$ and $d \in(0,1]$ such that for any $(x, y) \in \mathcal{K}^{2},\left(\theta_{1}, \theta_{2}\right) \in \Theta^{2}$ and $t \in R_{T}$,

$$
\left|\dot{S}_{\theta}^{3}\left(\theta_{1}, t, x\right)-\dot{S}_{\theta}^{3}\left(\theta_{2}, t, y\right)\right| \leq C_{\mathcal{K}}\left(|x-y|^{c}+\left|\theta_{1}-\theta_{2}\right|^{d}\right) .
$$

Now, we recall the existence and uniqueness result for solutions to equation (1) and some properties of the Green function which will be needed.

TheOREM 1. Under (H1) and (H2), there exists a unique continuous random field $X=\left\{X_{t}: t \in R_{T}\right\}$ which is a solution of (1).

Pro of. See Rovira and Sanz-Solé (1995), Proposition 2.1, or Farré and Nualart (1993), Theorem 2.1.

Lemma 2. Under (H1) and (H2), we have:

(i) For any $\theta \in \Theta$ and $t \in R_{T}$, the function $s \mapsto \gamma_{t}(\theta, s)$ has uniformly bounded derivatives of first order and mixed partial derivatives of second order on $\left\{s \in R_{T}: 0 \leq s^{1} \leq t^{1}, 0 \leq s^{2} \leq t^{2}\right\}$. 
(ii) There exists $C>0$ such that

$$
\sup _{\theta \in \Theta} \sup _{t \in R_{T}} \sup _{s \in R_{t}}\left|\gamma_{t}(\theta, s)\right| \leq C .
$$

Proof. This follows immediately from the boundedness of $S^{i}, i=1,2$, and techniques developed in the proofs of Propositions 3.1 and 3.2 of Rovira and Sanz-Solé (1995).

Lemma 3. There exists $C>0$ such that for any $t \in R_{T}$,

$$
\sup _{\theta \in \Theta}\left|\int_{R_{t}} \gamma_{t}(\theta, s) W(d s)\right| \leq C \sup _{s \in R_{t}}\left|W_{s}\right| .
$$

Proof. In view of Lemma 2(i), for any $\theta \in \Theta$ and $t \in R_{T}$, the function $s \mapsto \gamma_{t}(\theta, s)$ has uniformly bounded first order derivatives and mixed second order derivatives. Therefore, we have

$$
\begin{aligned}
\int_{R_{t}} \gamma_{t}(\theta, s) W(d s)= & W_{t}-\int_{0}^{t^{1}} \frac{\partial \gamma_{t}}{\partial s^{1}}\left(\theta, s^{1}, t^{2}\right) W_{s^{1}, t^{2}} d s^{1} \\
& -\int_{0}^{t^{2}} \frac{\partial \gamma_{t}}{\partial s^{2}}\left(\theta, t^{1}, s^{2}\right) W_{t^{1}, s^{2}} d s^{2}-\int_{R_{t}} \frac{\partial^{2} \gamma_{t}}{\partial s^{1} \partial s^{2}}(\theta, s) W_{s} d s .
\end{aligned}
$$

The desired result follows immediately.

Lemma 4. Under (H1) and (H2), there exists $C>0$ such that

$$
\sup _{\theta \in \Theta} \sup _{t \in R_{T}}\left|X_{t}(\theta)-x_{t}(\theta)\right| \leq C \varepsilon \sup _{t \in R_{T}}\left|W_{t}\right| .
$$

Proof. We have

$$
\begin{aligned}
\left|X_{s}(\theta)-x_{s}(\theta)\right| \leq & \int_{R_{s}}\left|\gamma_{s}(\theta, u)\left[S^{3}(\theta, u, X(\theta))-S^{3}(\theta, u, x(\theta))\right]\right| d u \\
& +\varepsilon\left|\int_{R_{s}} \gamma_{t}(\theta, u) W(d u)\right| .
\end{aligned}
$$

By Lemmas 2(ii) and 3, we have

$$
\left|X_{s}(\theta)-x_{s}(\theta)\right| \leq C \int_{R_{s}}\left|S^{3}(\theta, u, X(\theta))-S^{3}(\theta, u, x(\theta))\right| d u+\varepsilon C \sup _{u \in R_{s}}\left|W_{u}\right| .
$$

Let

$$
g_{s}=\sup _{\theta \in \Theta} \sup _{u \in R_{s}}\left|X_{u}(\theta)-x_{u}(\theta)\right| .
$$

In view of $(\mathrm{H} 2)$, we have

$$
g_{t} \leq C \int_{R_{t}} g_{u} d u+\varepsilon C \sup _{u \in R_{t}}\left|W_{u}\right|
$$


Now, by using the Gronwall Lemma (see Dozzi (1989), p. 91), we deduce that

$$
g_{t} \leq \varepsilon C \sup _{u \in R_{t}}\left|W_{u}\right| .
$$

Let $Y=\left\{Y_{t}: t \in R_{T}\right\}$ be the solution of the following stochastic partial differential equation:

$$
\frac{\partial^{2} Y_{t}}{\partial t^{1} \partial t^{2}}=S^{1}\left(\theta_{0}, t\right) \frac{\partial Y_{t}}{\partial t^{2}}+S^{2}\left(\theta_{0}, t\right) \frac{\partial Y_{t}}{\partial t^{1}}+\dot{S}_{x}^{3}\left(\theta_{0}, t, x_{t}\left(\theta_{0}\right)\right) Y_{t}+\dot{W}_{t}
$$

with $Y_{t}=0$ on the axes. We denote by $\widetilde{x}_{t}(\theta)$ the vector function of the derivatives of $x_{t}(\theta)$ with respect to $\theta$ and put

$$
J(\theta)=\int_{R_{T}} \widetilde{x}_{t}(\theta) \widetilde{x}_{t}^{\prime}(\theta) d \mu(t) .
$$

Let

$$
\xi=J\left(\theta_{0}\right)^{-1} \int_{R_{T}} Y_{t} \widetilde{x}_{t}\left(\theta_{0}\right) d \mu(t) \text { and } Z_{t}=\varepsilon^{-1}\left(X_{t}-x_{t}\left(\theta_{0}\right)\right) .
$$

Remark 5. $Y$ is a centered Gaussian random field. Therefore, $\xi$ is a centered Gaussian random variable with covariance matrix

$$
\Gamma=J\left(\theta_{0}\right)^{-1}\left[\int_{R_{T}} \int_{R_{T}} E\left(Y_{s} Y_{t}\right) \widetilde{x}_{t}\left(\theta_{0}\right) \widetilde{x}_{s}^{\prime}\left(\theta_{0}\right) d \mu(t) d \mu(s)\right] J\left(\theta_{0}\right)^{-1} .
$$

Lemma 6. Under (H1)-(H4), there exists $C>0$ such that

(i) $\sup _{t \in R_{T}}\left|Y_{t}\right| \leq C \sup _{t \in R_{T}}\left|W_{t}\right|$,

(ii) $\sup _{t \in R_{T}}\left|Z_{t}-Y_{t}\right| \leq C \varepsilon^{a} \sup _{t \in R_{T}}\left|W_{t}\right|^{1+a}$,

(iii) $\sup _{t \in R_{T}}\left|\widetilde{x}_{t}(\theta)-\widetilde{x}_{t}\left(\theta_{0}\right)\right|$

$$
\leq C\left(\left|\theta-\theta_{0}\right|+\left|\theta-\theta_{0}\right|^{a}+\left|\theta-\theta_{0}\right|^{b}+\left|\theta-\theta_{0}\right|^{c}+\left|\theta-\theta_{0}\right|^{d}\right) .
$$

P r o of. (i) In view of Lemma 2(ii), Lemma 3 and (H4), we have

$$
\sup _{s \in R_{t}}\left|Y_{s}\right| \leq C \int_{R_{t}}\left(\sup _{u \in R_{s}}\left|Y_{u}\right|\right) d u+C \sup _{u \in R_{t}}\left|W_{u}\right| .
$$

Now, the Gronwall Lemma leads to (i).

(ii) Let $g_{t}=\left|Z_{t}-Y_{t}\right|$. We have

$$
\begin{aligned}
g_{t} \leq & \int_{R_{t}}\left|\gamma_{t}\left(\theta_{0}, s\right)\right| \\
& \times\left|\varepsilon^{-1}\left[S^{3}\left(\theta_{0}, s, X_{s}\right)-S^{3}\left(\theta_{0}, s, x_{s}\left(\theta_{0}\right)\right)\right]-\dot{S}_{x}^{3}\left(\theta_{0}, s, x_{s}\left(\theta_{0}\right)\right) Y_{s}\right| d s .
\end{aligned}
$$


By Lemma 2(ii), we have

$$
g_{t} \leq C \int_{R_{t}}\left|\varepsilon^{-1}\left[S^{3}\left(\theta_{0}, s, X_{s}\right)-S^{3}\left(\theta_{0}, s, x_{s}\left(\theta_{0}\right)\right)\right]-\dot{S}_{x}^{3}\left(\theta_{0}, s, x_{s}\left(\theta_{0}\right)\right) Y_{s}\right| d s .
$$

Therefore, there exists $\tilde{X}_{s}=x_{s}\left(\theta_{0}\right)+\beta\left(X_{s}-x_{s}\left(\theta_{0}\right)\right), \beta \in(0,1)$, such that

$$
\begin{aligned}
g_{t} \leq & C \int_{R_{t}}\left|\varepsilon^{-1}\left(X_{s}-x_{s}\left(\theta_{0}\right)\right) \dot{S}_{x}^{3}\left(\theta_{0}, s, \widetilde{X}_{s}\right)-\dot{S}_{x}^{3}\left(\theta_{0}, s, x_{s}\left(\theta_{0}\right)\right) Y_{s}\right| d s \\
\leq & C \int_{R_{t}}\left|\left[\varepsilon^{-1}\left(X_{s}-x_{s}\left(\theta_{0}\right)\right)-Y_{s}\right] \dot{S}_{x}^{3}\left(\theta_{0}, s, \widetilde{X}_{s}\right)\right| d s \\
& +C \int_{R_{t}}\left|\left[\dot{S}_{x}^{3}\left(\theta_{0}, s, \widetilde{X}_{s}\right)-\dot{S}_{x}^{3}\left(\theta_{0}, s, x_{s}\left(\theta_{0}\right)\right)\right] Y_{s}\right| d s
\end{aligned}
$$

By using (H4), we obtain

$$
g_{t} \leq C \int_{R_{t}}\left(\sup _{u \in R_{s}} g_{u}\right) d s+C \int_{R_{t}}\left|\widetilde{X}_{s}-x_{s}\left(\theta_{0}\right)\right|^{a}\left|Y_{s}\right| d s .
$$

Now, by Lemma 4, we have

$$
\sup _{s \in R_{t}} g_{s} \leq C \int_{R_{t}}\left(\sup _{u \in R_{v}} g_{u}\right) d v+C \varepsilon^{a} \sup _{u \in R_{t}}\left|W_{u}\right|^{a} \sup _{u \in R_{t}}\left|Y_{u}\right| .
$$

In view of the Gronwall Lemma and (i), we deduce that

$$
\sup _{t \in R_{T}} g_{t} \leq C \varepsilon^{a} \sup _{t \in R_{T}}\left|W_{t}\right|^{1+a} .
$$

(iii) First of all, let us prove that for any $t \in R_{T}$ and $s \in R_{t}$, the function $\theta \mapsto \gamma_{t}(\theta, s)$ has uniformly bounded derivatives. To this end, recall that

$$
\gamma_{t}(\theta, s)=\sum_{n=0}^{\infty} H_{n}(\theta, t, s)
$$

where $H_{n}$ is defined by

$$
\begin{aligned}
H_{0}(\theta, s, t)= & 1 \\
H_{n+1}(\theta, s, t)= & \int_{s^{1}}^{t^{1}} S^{1}\left(\theta, u^{1}, s^{2}\right) H_{n}\left(\theta, t,\left(u^{1}, s^{2}\right)\right) d u^{1} \\
& +\int_{s^{2}}^{t^{2}} S^{2}\left(\theta, s^{1}, u^{2}\right) H_{n}\left(\theta, t,\left(s^{1}, u^{2}\right)\right) d u^{2}, \quad n \geq 0 .
\end{aligned}
$$

By using (H1) and an induction argument, one can prove that for any $n \geq 0$ 
and $s \in R_{t}$,

$$
\left|H_{n}(\theta, t, s)\right| \leq C^{n} \sum_{j=0}^{n}\left(\begin{array}{l}
n \\
j
\end{array}\right) \frac{\left(t^{1}-s^{1}\right)^{j}\left(t^{2}-s^{2}\right)^{n-j}}{j !(n-j) !} .
$$

Next, let us prove by induction that

$$
\left|\frac{\partial H_{n}}{\partial \theta}(\theta, t, s)\right| \leq n C^{n} \sum_{j=0}^{n}\left(\begin{array}{c}
n \\
j
\end{array}\right) \frac{\left(t^{1}-s^{1}\right)^{j}\left(t^{2}-s^{2}\right)^{n-j}}{j !(n-j) !} .
$$

For $n=0$, this is obvious. Now,

$$
\begin{aligned}
\frac{\partial H_{n+1}}{\partial \theta}(\theta, t, s)= & \int_{s^{1}}^{t^{1}} H_{n}\left(\theta, t,\left(u^{1}, s^{2}\right)\right) \dot{S}_{\theta}^{1}\left(\theta, u^{1}, s^{2}\right) d u^{1} \\
& +\int_{s^{1}}^{t^{1}} S^{1}\left(\theta, u^{1}, s^{2}\right) \frac{\partial H_{n}}{\partial \theta}\left(\theta, t,\left(u^{1}, s^{2}\right)\right) d u^{1} \\
& +\int_{s^{2}}^{t^{2}} H_{n}\left(\theta, t,\left(s^{1}, u^{2}\right)\right) \dot{S}_{\theta}^{2}\left(\theta, s^{1}, u^{2}\right) d u^{2} \\
& +\int_{s^{2}}^{t^{2}} S^{2}\left(\theta, s^{1}, u^{2}\right) \frac{\partial H_{n}}{\partial \theta}\left(\theta, t,\left(s^{1}, u^{2}\right)\right) d u^{2} .
\end{aligned}
$$

By using (H1), (H2), (2) and the induction hypothesis, we deduce that

$$
\begin{aligned}
\left|\frac{\partial H_{n+1}}{\partial \theta}(\theta, t, s)\right| \leq & (n+1) C^{n+1}\left[\int_{s^{1}}^{t^{1}} \sum_{j=0}^{n}\left(\begin{array}{c}
n \\
j
\end{array}\right) \frac{\left(u^{1}-s^{1}\right)^{j}\left(t^{2}-s^{2}\right)^{n-j}}{j !(n-j) !} d u^{1}\right. \\
& \left.+\int_{s^{2}}^{t^{2}} \sum_{j=0}^{n}\left(\begin{array}{c}
n \\
j
\end{array}\right) \frac{\left(t^{1}-s^{1}\right)^{j}\left(u^{2}-s^{2}\right)^{n-j}}{j !(n-j) !} d u^{2}\right] \\
= & (n+1) C^{n+1} \sum_{j=0}^{n+1}\left(\begin{array}{c}
n+1 \\
j
\end{array}\right) \frac{\left(t^{1}-s^{1}\right)^{j}\left(t^{2}-s^{2}\right)^{n+1-j}}{j !(n+1-j) !}
\end{aligned}
$$

and (3) is proved.

It follows that

$$
\left|\frac{\partial H_{n}}{\partial \theta}(\theta, t, s)\right| \leq n\left(2\left(T^{1}+T^{2}\right) C\right)^{n} \max _{j \in\{0, \ldots, n\}}\left(\frac{1}{j !}, \frac{1}{(n-j) !}\right) .
$$

Since $\max _{j \in\{0, \ldots, n\}}(1 / j !, 1 /(n-j) !)$ is equal to $((n / 2) !)^{-2}$ if $n$ is even, and to $(((n+1) / 2) !((n-1) / 2) !)^{-1}$ if $n$ is odd, we deduce that

$$
\sum_{n=0}^{\infty}\left|\frac{\partial H_{n}}{\partial \theta}(\theta, t, s)\right| \leq C<\infty
$$


which implies that $\theta \mapsto \gamma_{t}(\theta, s)$ is differentiable and

$$
\frac{\partial \gamma_{t}}{\partial \theta}(\theta, s)=\sum_{n=0}^{\infty} \frac{\partial H_{n}}{\partial \theta}(\theta, t, s) .
$$

Therefore

$$
\sup _{\theta \in \Theta} \sup _{t \in R_{T}} \sup _{s \in R_{t}}\left|\frac{\partial \gamma_{t}}{\partial \theta}(\theta, s)\right|<\infty .
$$

By (H3), (3) and noting that for any $(i, j) \in\{1, \ldots, k\}^{2}$,

$$
\begin{aligned}
\frac{\partial^{2} H_{n+1}}{\partial \theta_{i} \partial \theta_{j}}(\theta, t, s)= & \int_{s^{1}}^{t^{1}} \frac{\partial^{2} S^{1}}{\partial \theta_{i} \partial \theta_{j}}\left(\theta, u^{1}, s^{2}\right) H_{n}\left(\theta, t,\left(u^{1}, s^{2}\right)\right) d u^{1} \\
& +\int_{s^{1}}^{t^{1}} \frac{\partial S^{1}}{\partial \theta_{i}}\left(\theta, u^{1}, s^{2}\right) \frac{\partial H_{n}}{\partial \theta_{j}}\left(\theta, t,\left(u^{1}, s^{2}\right)\right) d u^{1} \\
& +\int_{s^{1}}^{t^{1}} \frac{\partial S^{1}}{\partial \theta_{j}}\left(\theta, u^{1}, s^{2}\right) \frac{\partial H_{n}}{\partial \theta_{i}}\left(\theta, t,\left(u^{1}, s^{2}\right)\right) d u^{1} \\
& +\int_{s^{1}}^{t^{1}} S^{1}\left(\theta, u^{1}, s^{2}\right) \frac{\partial^{2} H_{n}}{\partial \theta_{i} \partial \theta_{j}}\left(\theta, t,\left(u^{1}, s^{2}\right)\right) d u^{1} \\
& +\int_{s^{2}}^{t^{2}} \frac{\partial^{2} S^{2}}{\partial \theta_{i} \partial \theta_{j}}\left(\theta, s^{1}, u^{2}\right) H_{n}\left(\theta, t,\left(s^{1}, u^{2}\right)\right) d u^{2} \\
& +\int_{s^{2}}^{t^{2}} \frac{\partial S^{2}}{\partial \theta_{i}}\left(\theta, s^{1}, u^{2}\right) \frac{\partial H_{n}}{\partial \theta_{j}}\left(\theta, t,\left(s^{1}, u^{2}\right)\right) d u^{2} \\
& +\int_{s^{2}}^{t^{2}} \frac{\partial S^{2}}{\partial \theta_{j}}\left(\theta, s^{1}, u^{2}\right) \frac{\partial H_{n}}{\partial \theta_{i}}\left(\theta, t,\left(s^{1}, u^{2}\right)\right) d u^{2} \\
& +\int_{s^{2}}^{t^{2}} S^{2}\left(\theta, s^{1}, u^{2}\right) \frac{\partial^{2} H_{n}}{\partial \theta_{i} \partial \theta_{j}}\left(\theta, t,\left(s^{1}, u^{2}\right)\right) d u^{2},
\end{aligned}
$$

one can prove that for any $n \geq 0, \theta \in \Theta, t \in R_{T}$ and $s \in R_{T}$,

$$
\left|\frac{\partial^{2} H_{n}}{\partial \theta_{i} \partial \theta_{j}}(\theta, t, s)\right| \leq n(n+1) C^{n} \sum_{l=0}^{n}\left(\begin{array}{l}
n \\
l
\end{array}\right) \frac{\left(t^{1}-s^{1}\right)^{l}\left(t^{2}-s^{2}\right)^{n-l}}{l !(n-l) !} .
$$

It follows that

$$
\sup _{1 \leq i, j \leq k} \sup _{\theta \in \Theta} \sup _{t \in R_{T}} \sup _{s \in R_{t}}\left|\frac{\partial^{2} \gamma_{t}}{\partial \theta_{i} \partial \theta_{j}}(\theta, s)\right|<\infty .
$$


Now, let $\dot{\gamma}_{t, \theta}$ (resp. $\ddot{\gamma}_{t, \theta}$ ) stand for the vector (resp. matrix) function of the first (resp. second) order derivatives of $\gamma_{t}$ in $\theta$. We have

$$
\begin{aligned}
\widetilde{x}_{t}(\theta)= & \int_{R_{t}} S^{3}\left(\theta, s, x_{s}(\theta)\right) \dot{\gamma}_{t, \theta}(\theta, s) d s \\
& +\int_{R_{t}} \gamma_{t}(\theta, s)\left[\dot{S}_{\theta}^{3}\left(\theta, s, x_{s}(\theta)\right)+\dot{S}_{x}^{3}\left(\theta, s, x_{s}(\theta)\right) \widetilde{x}_{s}(\theta)\right] d s .
\end{aligned}
$$

Therefore

$$
\begin{aligned}
\left|\widetilde{x}_{t}(\theta)-\widetilde{x}_{t}\left(\theta_{0}\right)\right| \leq & \int_{R_{t}}\left|\left[S^{3}\left(\theta, s, x_{s}(\theta)\right)-S^{3}\left(\theta_{0}, s, x_{s}\left(\theta_{0}\right)\right)\right] \dot{\gamma}_{t, \theta}(\theta, s)\right| d s \\
& +\int_{R_{t}}\left|S^{3}\left(\theta_{0}, s, x_{s}\left(\theta_{0}\right)\right)\left[\dot{\gamma}_{t, \theta}(\theta, s)-\dot{\gamma}_{t, \theta}\left(\theta_{0}, s\right)\right]\right| d s \\
& +\int_{R_{t}}\left|\gamma_{t}(\theta, s)\left[\dot{S}_{\theta}^{3}\left(\theta, s, x_{s}(\theta)\right)-\dot{S}_{\theta}^{3}\left(\theta_{0}, s, x_{s}\left(\theta_{0}\right)\right)\right]\right| d s \\
& +\int_{R_{t}}\left|\left[\gamma_{t}(\theta, s)-\gamma_{t}\left(\theta_{0}, s\right)\right] \dot{S}_{\theta}^{3}\left(\theta_{0}, s, x_{s}\left(\theta_{0}\right)\right)\right| d s \\
& +\int_{R_{t}}\left|\gamma_{t}(\theta, s) \dot{S}_{x}^{3}\left(\theta, s, x_{s}(\theta)\right)\left(\widetilde{x}_{s}(\theta)-\widetilde{x}_{s}\left(\theta_{0}\right)\right)\right| d s \\
& +\int_{R_{t}}\left|\gamma_{t}(\theta, s) \widetilde{x}_{s}\left(\theta_{0}\right)\left[\dot{S}_{x}^{3}\left(\theta, s, x_{s}(\theta)\right)-\dot{S}_{x}^{3}\left(\theta_{0}, s, x_{s}\left(\theta_{0}\right)\right)\right]\right| d s \\
& +\int_{R_{t}}\left|\dot{S}_{x}^{3}\left(\theta_{0}, s, x_{s}\left(\theta_{0}\right)\right) \widetilde{x}_{s}\left(\theta_{0}\right)\left[\gamma_{t}(\theta, s)-\gamma_{t}\left(\theta_{0}, s\right)\right]\right| d s .
\end{aligned}
$$

By using (H1)-(H4), the boundedness of $\dot{\gamma}_{t, \theta}, \ddot{\gamma}_{t, \theta}$ and noting that the function $(\theta, t) \mapsto x_{t}(\theta)$ is bounded, we deduce that

$$
\begin{aligned}
\left|\widetilde{x}_{t}(\theta)-\widetilde{x}_{t}\left(\theta_{0}\right)\right| & \\
\leq & C\left(\left|\theta-\theta_{0}\right|+\left|\theta-\theta_{0}\right|^{b}+\left|\theta-\theta_{0}\right|^{d}+\int_{R_{t}}\left|x_{s}(\theta)-x_{s}\left(\theta_{0}\right)\right| d s\right. \\
& +\int_{R_{t}}\left|x_{s}(\theta)-x_{s}\left(\theta_{0}\right)\right|^{a} d s+\int_{R_{t}}\left|x_{s}(\theta)-x_{s}\left(\theta_{0}\right)\right|^{c} d s \\
& \left.+\int_{R_{t}}\left|\widetilde{x}_{s}(\theta)-\widetilde{x}_{s}\left(\theta_{0}\right)\right| d s\right) .
\end{aligned}
$$

Now, it is not difficult to see that the function $(\theta, t) \mapsto \widetilde{x}_{t}(\theta)$ is bounded. So,

$$
\sup _{t \in R_{T}}\left|x_{t}(\theta)-x_{t}\left(\theta_{0}\right)\right| \leq\left|\theta-\theta_{0}\right| .
$$


Hence, from (4) and the Gronwall Lemma, we deduce that

$\sup _{t \in R_{T}}\left|\widetilde{x}_{t}(\theta)-\widetilde{x}_{t}\left(\theta_{0}\right)\right| \leq C\left(\left|\theta-\theta_{0}\right|+\left|\theta-\theta_{0}\right|^{a}+\left|\theta-\theta_{0}\right|^{b}+\left|\theta-\theta_{0}\right|^{c}+\left|\theta-\theta_{0}\right|^{d}\right)$.

Now, since the ingredients for the proofs of the main results are assembled, we can deal with the asymptotic behavior of the estimator $\theta_{\varepsilon}^{*}$.

3. Results. Let $g_{\theta_{0}}(\delta)=\inf _{\left|\theta-\theta_{0}\right|>\delta}\left\|x(\theta)-x\left(\theta_{0}\right)\right\|_{L^{2}(\mu)}$ and $g(\delta)=$ $\inf _{\theta_{0} \in K} g_{\theta_{0}}(\delta)$ where $K$ is an arbitrary compact subset of $\Theta$. The following theorem ensures the consistency of $\theta_{\varepsilon}^{*}$.

Theorem 7. Under (H1) and (H2), there exists a constant $C>0$ (independent of $K)$ such that for any $\delta>0$ and $\varepsilon \in(0,1]$,

$$
\sup _{\theta_{0} \in K} P_{\theta_{0}}^{(\varepsilon)}\left(\left|\theta_{\varepsilon}^{*}-\theta_{0}\right| \geq \delta\right) \leq C \exp \left(-\frac{g^{2}(\delta)}{\varepsilon^{2} C}\right) .
$$

Proof. The proof is similar to that of Kutoyants and Lessi (1995), Theorem 3.1, and uses Theorem 1 and an exponential inequality for the Wiener random field.

The next result concerns the asymptotic law of $\theta_{\varepsilon}^{*}$ as $\varepsilon \rightarrow 0$.

Theorem 8. Assume that (H1)-(H4) are satisfied and for any $\delta>0$,

$$
g(\delta)>0, \quad \inf _{|u|=1}\left\langle J\left(\theta_{0}\right) u, u\right\rangle>0 .
$$

Then

$$
P_{\theta_{0}}-\lim _{\varepsilon \rightarrow 0} \varepsilon^{-1}\left(\theta_{\varepsilon}^{*}-\theta_{0}\right)=\xi
$$

where $P_{\theta_{0}}$ - lim denotes the convergence with respect to the probability $P_{\theta_{0}}$.

Proof. The proof is essentially based on Lemma 6 and follows the same lines as that of Kutoyants and Lessi (1995), Theorem 4.3. So, we only point out the minor adaptations needed. We make the change of variable $u=\varepsilon^{-1}\left(\theta-\theta_{0}\right)$ and put

$$
\begin{gathered}
U_{\theta_{0}, \varepsilon}=\left\{u \in \mathbb{R}^{k}: \theta_{0}+\varepsilon u \in \Theta\right\}, \\
u_{\varepsilon}^{*}=\arg \inf _{u \in U_{\theta_{0}, \varepsilon}}\left\|Z-\frac{x\left(\theta_{0}+\varepsilon u\right)-x\left(\theta_{0}\right)}{\varepsilon}\right\|_{L^{2}(\mu)} .
\end{gathered}
$$

We have $u_{\varepsilon}^{*}=\varepsilon^{-1}\left(\theta_{\varepsilon}^{*}-\theta_{0}\right)$.

Now, set

$$
A_{1}=\left\{\omega \in \Omega:\left\|X-x\left(\theta_{0}\right)\right\|_{L^{2}(\mu)}<\inf _{\substack{u \in U_{\theta_{0}, \varepsilon} \\|u|>\lambda_{\varepsilon}}}\left\|X-x\left(\theta_{0}+\varepsilon u\right)\right\|_{L^{2}(\mu)}\right\}
$$


where $\lambda_{\varepsilon}=\varepsilon^{-\delta}, \delta \in(0,1]$. Following Kutoyants and Lessi (1995), we have

$$
u_{\varepsilon}^{*}=J\left(\theta_{0}\right)^{-1} \int_{R_{T}} Z_{t} \widetilde{x}_{s}\left(\theta_{\varepsilon}^{*}\right) d \mu(t)-J\left(\theta_{0}\right)^{-1}\left[J\left(\theta_{\varepsilon}, \theta_{\varepsilon}^{*}\right)^{\prime}-J\left(\theta_{0}\right)\right] u_{\varepsilon}^{*} .
$$

Therefore

$$
\begin{aligned}
\left|u_{\varepsilon}^{*}-\xi\right| \leq & \left|J\left(\theta_{0}\right)^{-1}\left[\int_{R_{T}}\left(Z_{t}-Y_{t}\right) \widetilde{x}_{t}\left(\theta_{\varepsilon}^{*}\right) d \mu(t)+\int_{R_{T}}\left(\widetilde{x}_{t}\left(\theta_{\varepsilon}^{*}\right)-\widetilde{x}_{t}\left(\theta_{0}\right)\right) Y_{t} d \mu(t)\right]\right| \\
& +\left|J\left(\theta_{0}\right)^{-1}\left[J\left(\theta_{\varepsilon}, \theta_{\varepsilon}^{*}\right)^{\prime}-J\left(\theta_{0}\right)\right] u_{\varepsilon}^{*}\right|,
\end{aligned}
$$

where $J\left(\theta_{\varepsilon}, \theta_{\varepsilon}^{*}\right)^{\prime}$ is equal to the matrix $\int_{R_{t}} \widetilde{x}_{t}\left(\theta_{\varepsilon}^{*}\right) \widetilde{x}_{t}^{\prime}\left(\theta_{\varepsilon}\right) d \mu(t)$ and $\theta_{\varepsilon}=\theta_{0}+$ $\gamma_{t} \varepsilon u, \gamma_{t} \in[0,1)$. By Lemma 6 , on $A_{1}$ we have

$$
\begin{aligned}
\left|u_{\varepsilon}^{*}-\xi\right| \leq & C\left(\varepsilon^{a} \sup _{t \in R_{T}}\left|W_{t}\right|^{1+a}+\varepsilon^{1-\delta}+\varepsilon^{a-\delta}+\varepsilon^{b-\delta}+\varepsilon^{c-\delta}+\varepsilon^{d-\delta}\right) \\
& +C\left(\varepsilon^{1-\delta}+\varepsilon^{a(1-\delta)}+\varepsilon^{b(1-\delta)}+\varepsilon^{c(1-\delta)}+\varepsilon^{d(1-\delta)}\right) \sup _{t \in R_{T}}\left|W_{t}\right| .
\end{aligned}
$$

Now, put $e=a \wedge b \wedge c \wedge d$. Since $\varepsilon \in[0,1)$, we deduce that on $A_{1}$ we have

$$
\left|u_{\varepsilon}^{*}-\xi\right| \leq C\left(\varepsilon^{a} \sup _{t \in R_{T}}\left|W_{t}\right|^{1+a}+\varepsilon^{e-\delta}+\varepsilon^{e(1-\delta)} \sup _{t \in R_{T}}\left|W_{t}\right|\right) .
$$

Let $r=a \wedge(e-\delta)$ and choose $\delta=e /(1+a)$. Then on $A_{1}$ we have

$$
\left|u_{\varepsilon}^{*}-\xi\right| \leq C \varepsilon^{r / 2}\left(\varepsilon^{a-r / 2} \sup _{t \in R_{T}}\left|W_{t}\right|^{1+a}+\varepsilon^{e-\delta-r / 2}+\varepsilon^{e(1-\delta)-r / 2} \sup _{t \in R_{T}}\left|W_{t}\right|\right) .
$$

Now, set

$$
A_{2}=\left\{\omega \in \Omega: \sup _{t \in R_{T}}\left|W_{t}(\omega)\right|<\varepsilon^{-\varrho}\right\} \quad \text { and } \quad A=A_{1} \cap A_{2}
$$

where $\varrho=\min \left\{(a-r / 2)(1+a)^{-1}, e-\delta-r / 2\right\}$. Then on $A$ we have

$$
\left|u_{\varepsilon}^{*}-\xi\right| \leq C \varepsilon^{r / 2}\left(\varepsilon^{\alpha_{1}}+\varepsilon^{\alpha_{2}}+\varepsilon^{\alpha_{3}}\right)
$$

where

$\alpha_{1}=a-\varrho(1+a)-r / 2 \geq 0, \alpha_{2}=e-\delta-r / 2 \geq 0, \alpha_{3}=e-\delta-\varrho-r / 2 \geq 0$.

Following Kutoyants and Lessi (1995), one can prove that $P_{\theta_{0}}^{(\varepsilon)}(\bar{A}) \rightarrow 0$ as $\varepsilon \rightarrow 0$ where $\bar{A}=\Omega \backslash A$, which completes the proof.

Now, let us apply the above results to the two-parameter OrnsteinUlhenbeck process with parameter $\left(\theta^{1}, \theta^{2}, \varepsilon\right)$ which is a random field $H$ defined in Dozzi (1989), p. 155, by

$$
H_{t}=e^{\theta^{1} t^{1}} x+e^{\theta^{2} t^{2}} x-e^{\theta^{\prime} t} x+\varepsilon e^{\theta^{\prime} t} \int_{R_{T}} e^{-\theta^{\prime} u} x d W_{u} .
$$

We remark that $H_{t}=x$ on the axes. Itô's formula in Guyon and Prum (1981), p. 634, implies that $H$ satisfies the nonlinear hyperbolic stochastic 
partial differential equation

$$
\frac{\partial^{2} H_{t}}{\partial t^{1} \partial t^{2}}=\theta^{1} \frac{\partial H_{t}}{\partial t^{2}}+\theta^{2} \frac{\partial H_{t}}{\partial t^{1}}-\theta^{1} \theta^{2} H_{t}+\varepsilon \dot{W}_{t}, \quad t \in \mathbb{R}_{+}^{2} .
$$

Assume that $\theta=\left(\begin{array}{c}\theta^{1} \\ \theta^{2}\end{array}\right)$ is unknown, $\theta \in \Theta$, where $\Theta$ is a bounded open subset of $\mathbb{R}^{2}$, and put

$$
\theta_{\varepsilon}^{* *}=\arg \inf _{\theta \in \Theta}\|H-x(\theta)\|_{L^{2}(\mu)}
$$

where

$$
x_{t}(\theta)=e^{\theta^{1} t^{1}} x+e^{\theta^{2} t^{2}} x-e^{\theta^{\prime} t} x
$$

We have

Corollary 9. (i) There exists $C>0$ (independent of the compact set $K$ ) such that

$$
\sup _{\theta_{0} \in K} P_{\theta_{0}}^{(\varepsilon)}\left(\left|\theta_{\varepsilon}^{* *}-\theta_{0}\right| \geq \delta\right) \leq \exp \left(-\frac{g^{2}(\delta)}{\varepsilon^{2} C}\right) .
$$

(ii) $P_{\theta_{0}}-\lim _{\varepsilon \rightarrow 0} \varepsilon^{-1}\left(\theta_{\varepsilon}^{* *}-\theta_{0}\right)=\xi$ where $\xi$ is a centered Gaussian random variable with covariance matrix

$$
\begin{aligned}
\Gamma= & J\left(\theta_{0}\right)^{-1}\left[\int_{R_{T}} \int_{R_{T}} \frac{1}{\theta_{0}^{1} \theta_{0}^{2}}\left(e^{\theta_{0}^{1}\left|t^{1}-s^{1}\right|}-e^{\theta_{0}^{1}\left(t^{1}+s^{1}\right)}\right)\right. \\
& \left.\times\left(e^{\theta_{0}^{2}\left|t^{2}-s^{2}\right|}-e^{\theta_{0}^{2}\left(t^{2}+s^{2}\right)}\right) \widetilde{x}_{t}\left(\theta_{0}\right) \widetilde{x}_{s}^{\prime}\left(\theta_{0}\right) d \mu(t) d \mu(s)\right] J\left(\theta_{0}\right)^{-1} .
\end{aligned}
$$

Pr o of. It suffices to verify that in this case (H1)-(H4) are satisfied and

$$
\begin{gathered}
Y_{t}=e^{\theta_{0}^{\prime} t} \int_{R_{t}} e^{-\theta_{0}^{\prime} u} d W_{u} \\
E\left(Y_{t} Y_{s}\right)=\frac{1}{\theta_{0}^{1} \theta_{0}^{2}}\left(e^{\theta_{0}^{1}\left|t^{1}-s^{1}\right|}-e^{\theta_{0}^{1}\left(t^{1}+s^{1}\right)}\right)\left(e^{\theta_{0}^{2}\left|t^{2}-s^{2}\right|}-e^{\theta_{0}^{2}\left(t^{2}+s^{2}\right)}\right) .
\end{gathered}
$$

Then use Remark 5 and apply Theorems 7 and 8.

\section{References}

H. Dietz and Y. Kutoyants (1992), A minimum-distance estimator for diffusion processes with ergodic properties, Tech. Report 11, Inst. Appl. Analysis and Stochastics, Berlin.

H. Dietz and Y. Kutoyants (1997), A class of minimum-distance estimators for diffusion processes with ergodic properties, Statistics and Decisions 15, 211-217.

M. Dozzi (1989), Stochastic Processes with a Multidimensional Parameter, Longman Sci. Tech.

M. Farré and D. Nualart (1993), Nonlinear stochastic integral equations in the plane, Stochastic Process. Appl. 46, 219-239. 
X. Guyon and B. Prum (1981), Semimartingales à deux indices, Ph.D. Thesis, Univ. de Paris VI.

S. Hénaff (1995), On minimum distance estimate of the parameter of the OrnsteinUhlenbeck process, preprint, Univ. of Angers.

H. Korezlioglu, G. Mazziotto and J. Szpirglas (1983), Nonlinear filtering equations for two parameter semimartingales, Stochastic Process. Appl. 15, 239-269.

Y. Kutoyants (1994), Identification of Dynamical Systems with Small Noise, Kluwer, Dordrecht.

Y. Kutoyants and O. Lessi (1995), Minimum distance estimation for diffusion random fields, Publ. Inst. Statist. Univ. Paris 29, fasc. 3, 3-20.

Y. Kutoyants, A. Nercessian and P. Pilibossian (1994), On limit distribution of the minimum sup norm estimate of the parameter of the Ornstein-Uhlenbeck process, Romanian J. Pure Appl. Math. 39, 119-139.

Y. Kutoyants and P. Pilibossian (1994), On minimum $L_{1}$ estimate of the parameter of the Ornstein-Uhlenbeck process, Statist. Probab. Lett. 20, 117-123.

J. Norris (1995), Twisted sheets, J. Funct. Anal. 132, 273-334.

C. Rovira and M. Sanz-Solé (1995), A nonlinear hyperbolic SPDE: Aproximations and support, in: London Math. Soc. Lecture Note Ser. 216, Cambridge Univ. Press, $241-261$.

C. Rovira and M. Sanz-Solé (1996), The law of the solution to a nonlinear hyperbolic SPDE, J. Theoret. Probab. 9, 863-901.

Vincent Monsan and Modeste N'zi

Université de Cocody

UFR de Mathématiques et Informatique

Equipe de Probabilités et Statistique

BP 582 Abidjan 22, Côte d'Ivoire

E-mail: monsanv@ci.refer.org

nziy@ci.refer.org 